\title{
Situación actual de estándares e.Learning y aplicación en entornos de Software Libre
}

Juan Lago Cabrera.

Fundación IAVANTE. Consejería de Salud de Andalucía.

Esta monografía trata de presentar un breve informe de situación de los estándares e.Learning con especial mención a SCORM, estándar "de facto" que está cobrando bastante importancia en los últimos años. Entre los objetivos que se persiguen con estos estándares, está la reutilización de contenidos de aprendizaje con independencia de la tecnología de las herramientas que los generan (herramientas de autor) y los usan (plataformas e.Learning). Son muchas las empresas, instituciones y organizaciones educativas que están adoptando SCORM, pero es interesante ver cómo también la comunidad de Software Libre ha sabido adaptarse a la evolución de estos estándares, pudiéndose encontrar en Internet multitud de herramientas que los soportan. Como ejemplo práctico, se verá cómo integrar un contenido SCORM dentro de una plataforma LMS de Software Libre como Moodle así como una breve descripción de algunas herramientas de autor que se pueden encontrar en la Web.

Correspondencia:

J. Lago

Fundación IAVANTE

c/Hermes 10,3-2B

e-mail: juan.lago@iavante.es

\section{INTRODUCCIÓN (A LOS ESTÁNDARES E.LEARNING)}

La industria e.Learning continúa su expansión día a día lo que provoca la aparición de nuevas especificaciones, guías o normas por parte de la comunidad internacional que intentan conseguir varios objetivos fundamentales: mejorar la formación mediante la utilización de contenidos de aprendizaje de calidad, identificar y aplicar mejores prácticas y la reutilización de contenidos con independencia de la tecnología o plataforma LMS (Learning Management System o Sistema de Gestión del Aprendizaje) concreta.

Esta reutilización debe ser entendida de forma amplia cubriendo los siguientes aspectos:

- Poder mover contenidos de aprendizaje de un LMS a otro.

- Poder reutilizar parte de los contenidos de un curso en otros cursos.

- Poder acceder y utilizar repositorios de contenidos de aprendizaje, algo así como una biblioteca de contenidos remota.

Cuando estas especificaciones son acreditadas por organizaciones internacionales reconocidas 
(como por ejemplo IEEE), se convierten en estándares. A continuación se resume brevemente las organizaciones y estándares más importantes hoy día que están trabajando en el ámbito e.Learning:

Aviation Industry CBT Committee (AICC) (a): es una asociación internacional de profesionales de la formación basada en medios tecnológicos (CBT, Computer Based Training). AICC elabora guías para la industria de la aviación en el desarrollo, entrega y evaluación de tecnologías aplicadas a la formación. Sus objetivos son conseguir una formación más rentable, eficiente y sostenible. De entre todas sus recomendaciones, la guía CMI (Computer-Managed Instruction) es la que ha tenido más impacto.

IMS Global Consortium( ${ }^{(b)}$ : organización internacional sin ánimo de lucro que desarrolla y promueve la adopción de especificaciones técnicas abiertas para conseguir tecnologías de aprendizaje interoperables. Entre sus miembros se encuentran agencias gubernamentales, fabricantes de hardware y software, instituciones educativas, integradores de sistemas, proveedores de contenidos multimedia, etc. Uno de los puntos en los que se centra IMS es en la especificación basada en XML (eXtensible Markup Language) aplicada a cursos, lecciones, evaluaciones, estudiantes y grupos. Las especificaciones de IMS más conocidas son IMS Meta-data, IMS Content Packaging y IMS QTI (Question and Test Interchange).

Institute for Electrical and Electronic Engineers Learning Technology Standards Committee (IEEE LTSC)( $\left.{ }^{(}\right)$: organización internacional que desarrolla estándares técnicos y recomendaciones para sistemas eléctricos, electrónicos, informáticos y de comunicaciones. Dentro de ella, el comité LTSC se centra en el ámbito de las tecnologías de aprendizaje. La especificación más ampliamente reconocida elaborado por IEEE LTSC es LOM (Learning Object Metadata), la cual define grupos de elementos y elementos que describen a los recursos de aprendizaje. Tanto IMS como ADL han adoptado LOM dentro de sus especificaciones.

Advanced Distributed Learning (ADL) $\left.{ }^{(}\right)$: es una iniciativa creada por el gobierno de Estados Unidos que investiga y desarrolla especificaciones para fomentar el avance del e.Learning. ADL emplea el esfuerzo colaborativo de sectores públicos y privados para desarrollar estándares, herramientas y contenidos de aprendizaje de calidad. La visión de ADL es permitir el acceso a materiales de formación de alta calidad adaptado a las necesidades individuales y que sean fácilmente accesibles. La especificación más ampliamente aceptada de ADL es SCORM (Shareable Content Object Referente Model) de la cual se hablará un poco más adelante en este artículo. Una característica importante a destacar de SCORM es que combina elementos de las especificaciones aportadas por las tres organizaciones anteriores (AICC, IMS e IEEE).

Hay que destacar que las tres principales áreas en las que trabajan todas las especificaciones anteriores y las organizaciones que la soportan son:

- El empaquetado de contenidos formativos, es decir, cómo agrupar los recursos en un formato manejable.

- Los metadatos o cómo describir de una manera consistente los contenidos de aprendizaje. El propósito y utilidad de los metadatos es dotar a los contenidos de una información rica que permita encontrar, ensamblar y entregar el contenido de aprendizaje adecuado a cada público objetivo y en cada proceso de enseñanza-aprendizaje concreto.

- Interfaz de comunicación o API (Application Program Interface), define cómo los recursos de aprendizaje se comunican con la plataforma para intercambiar información dinámicamente como por ejemplo para indicar que un alumno ha completado un tema o la nota obtenida en la realización de un ejercicio. Sólo los estándares promovidos por AICC y ADL hacen referencia a este interfaz de comunicación.

Hay que aclarar que la interfaz de comunicación o API afecta tanto a las plataformas LMS como a los contenidos dado que, como se acaba de comentar, define la forma en que se comunican ambos elementos. Los fabricantes de Software pueden acudir a la documentación existente sobre requisitos de conformidad para certificar sus soluciones.

Dada la importancia que la especificación SCORM ha tenido en los últimos tiempos y por su cualidad de aglutinador de otros estándares, a continuación se va a comentar brevemente en qué consiste.

\section{ADL SCORM}

SCORM se ha convertido en un estándar "de facto", es decir, no ha sido acreditado por organismos internacionales pero existe una masa crítica de proyectos, empresas e iniciativas que lo han adoptado. SCORM no es una aplicación Software ni tampoco trata la calidad de los contenidos, sino de las caracte- 
rísticas necesarias para que el contenido pueda ser reutilizado dentro de un entorno técnico para el aprendizaje basado en Web (WBT, Web-based Training).

SCORM es un conjunto de especificaciones que describen:

- Cómo crear contenidos e.Learning basados en Web que se puedan importar en plataformas e.Learning que cumplan SCORM y que se pueda realizar un seguimiento de dichos contenidos.

- Qué tiene que cumplir un LMS con el objeto de poder importar y realizar seguimiento de un contenido que cumpla SCORM.

Para conseguir estos objetivos, SCORM se centra en dos aspectos fundamentales que afectan a los objetos de aprendizaje:

- Content Aggregation Model o Modelo de Agrupación de Contenidos: que se centra en identificar las unidades de contenidos básicas (como puede ser un archivo de video, un archivo Flash, una imagen JPG, un archivo HTML, archivos de texto, etc) y la manera en que se agrupan hasta obtener una estructura completa de un tema, una unidad didáctica o un curso completo.

- Run-time Environment o Entorno de Ejecución: que se centra en definir cómo los contenidos se comunican con la plataforma LMS de forma que no dependa de un fabricante concreto o de la manera en que se hayan generado esos contenidos.

La figura 1 esquematiza el conjunto de recomendaciones sobre las que se apoya SCORM, en la que se puede apreciar la reutilización de especificaciones de otros organismos internacionales como los que se comentaron en la introducción de este artículo.

Figura 1. SCORM como conjunto de estándares

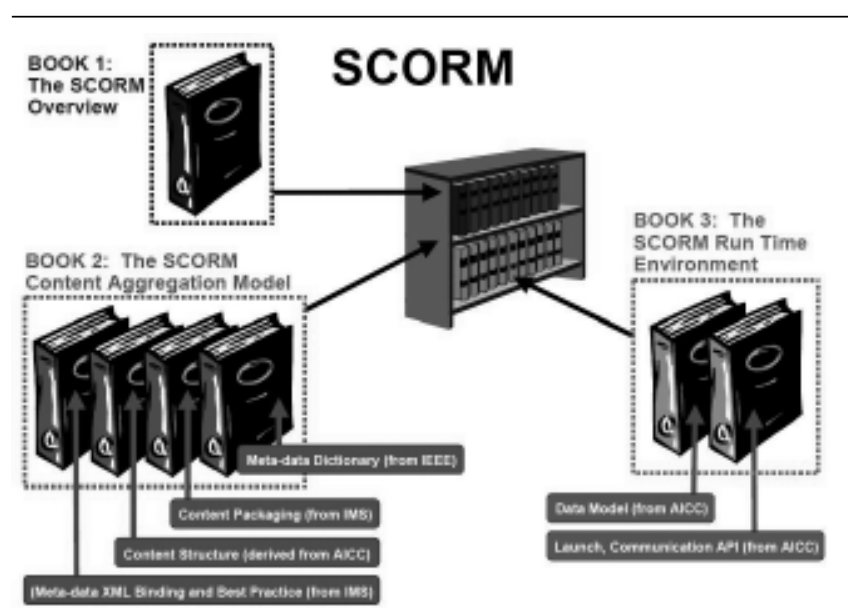

SCORM ha ido evolucionando a lo largo del tiempo y fruto de esta evolución ha sido la aparición de distintas versiones en sus especificaciones. Muchas de ellas frutos de mejoras y aportaciones de la comunidad y otras por evoluciones de los estándares subyacentes:

- En Enero del año 2000 entró en fase de pruebas la versión 1.0 de SCORM. Como se esperaba, los participantes y colaboradores de la comunidad de ADL plantearon una serie de dudas y cuestiones que propiciaron la rápida aparición de la versión 1.1

- En Octubre del año 2001, aparece la versión 1.2 de SCORM, una de las versiones más ampliamente soportadas en la actualidad tanto por las herramientas Software de generación de contenidos como por las plataformas e.Learning. La figura 1 corresponde al diagrama de estándares de SCORM 1.2.

- Posteriormente surgió SCORM 2004 (o también conocida como la versión 1.3) de la que han surgido varias ediciones. Recientemente se acaba de publicar la tercera edición. Se espera que SCORM 2004 3rd Edition sea una evolución estable de SCORM en la que no se prevé grandes cambios en el futuro. SCORM 2004 viene a corregir algunas deficiencias de la versión anterior, la 1.2, sobre todo en cuanto a posibilidades secuenciación y navegación en los contenidos, es decir, qué contenidos se muestran en cada momento en función de los criterios pedagógicos definidos por el diseñador de los mismos.

Uno de los puntos fuertes de esta evolución es que el estándar se va haciendo maduro y estable y va ampliando potencialidades aunque tiene el inconveniente de la diversidad de implementaciones existentes en las herramientas que se pueden encontrar en la Web o las razonables dudas de cuándo realizar un cambio de versión en las implementaciones del estándar.

\section{Un ejercicio práctico: carga y utilización de un curso SCORM en una plataforma LMS de Software Libre}

A continuación se va a realizar una breve demostración práctica de cómo cargar un contenido SCORM en una plataforma LMS de Software Libre que también cumpla el estándar SCORM.

La plataforma elegida ha sido Moodle(e), un Sistema de Gestión de Cursos (CMS, Course Management System) de Software Libre que está cobrando mucha importancia últimamente y que está siendo utilizada por una amplia comunidad de usua- 
Figura 2. Creación de un curso SCORM en Moodle

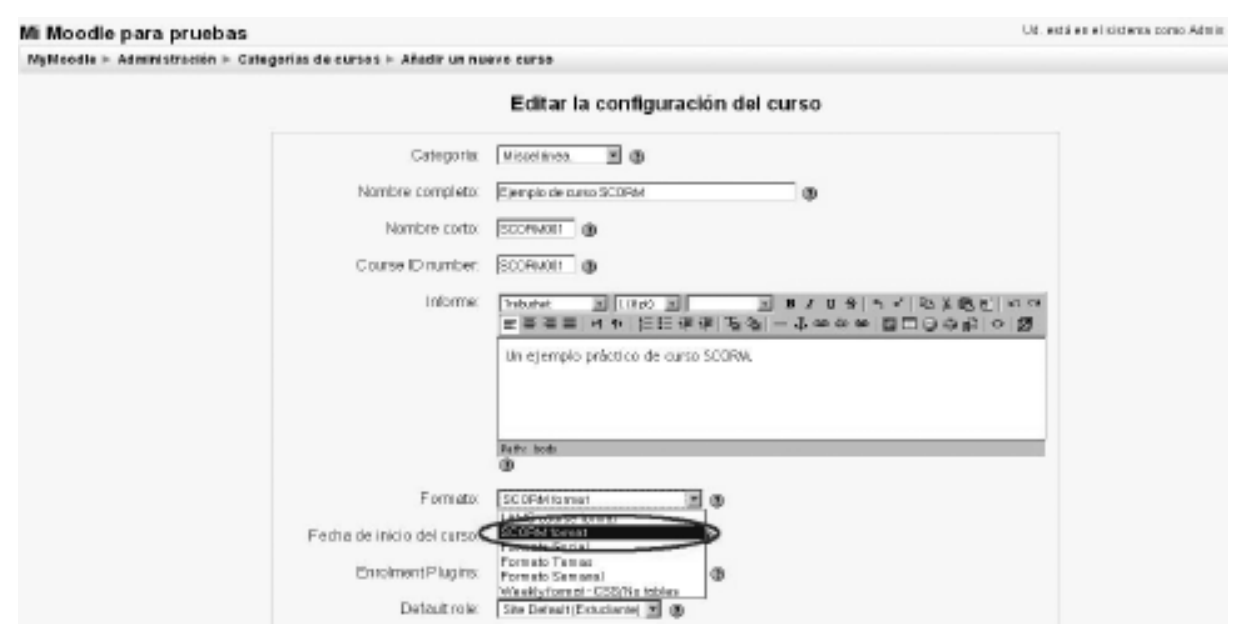

Figura 3. Carga del paquete SCORM en Moodle

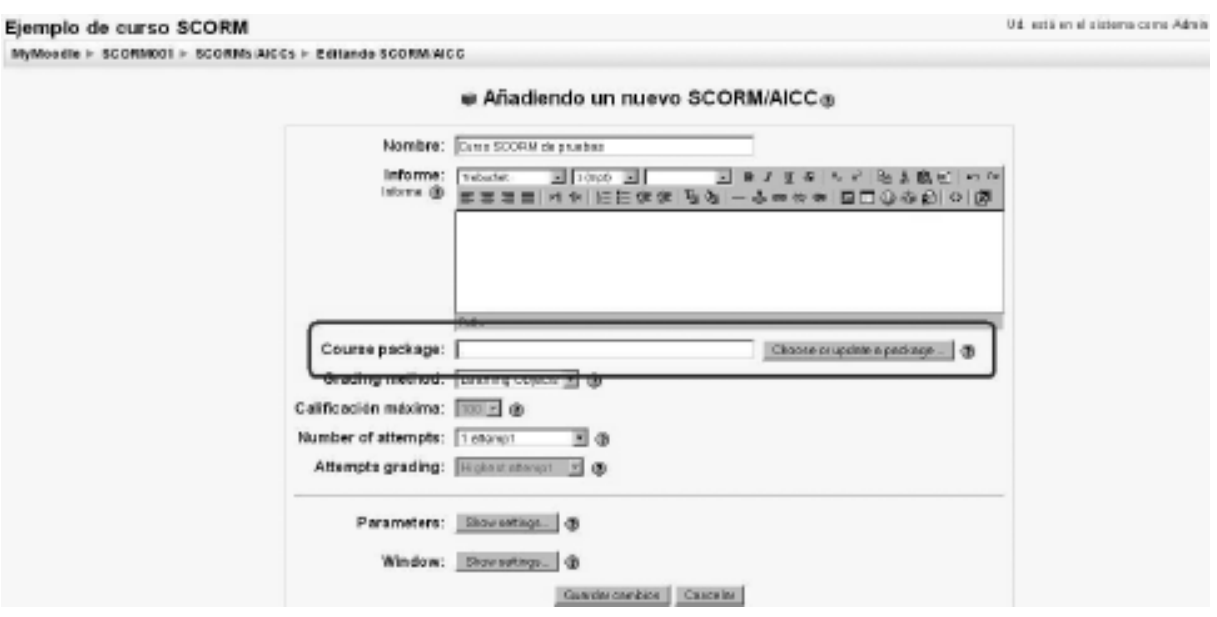

rios y organizaciones docentes. El portal Moodle cuenta con alrededor de 150.000 usuarios registrados, más de 18.000 instalaciones conocidas, con una media que supera las 40.000 descargas mensuales.

Moodle soporta SCORM 1.2 desde la versión 1.5 (Junio 2005) y SCORM 2004 desde la versión 1.5.3 (Noviembre 2005). Para esta demostración práctica, se ha utilizado la versión 1.7 de Moodle liberada recientemente (Noviembre 2006) que ha sido instalada sobre un servidor Linux. Es importante destacar que Moodle es capaz de importar y comunicarse con cursos en formato SCORM pero no es capaz de generar paquetes SCORM (Moodle no es una herramienta de autor). De esto se encargan las herra- mientas de generación de contenidos, de las cuales veremos algunos ejemplos en el siguiente apartado.

Una vez que se accede a la plataforma como administrador, Moodle permite crear un nuevo tipo de curso cuyo formato es SCORM (ver figura 2). Después de crear el curso, la plataforma solicita cuál es el paquete SCORM (figura 3) con el que se quiere trabajar, que normalmente consta de un fichero en formato ZIP (comprimido), el cual contiene toda la información de la estructura del curso y así como todos los recursos que utiliza (imágenes, páginas HTML, vídeos, audio, etc). Este fichero debe ser "subido" al servidor mediante el gestor de archivos que trae Moodle. 
Figura 4. Estructura de un curso SCORM en Moodle

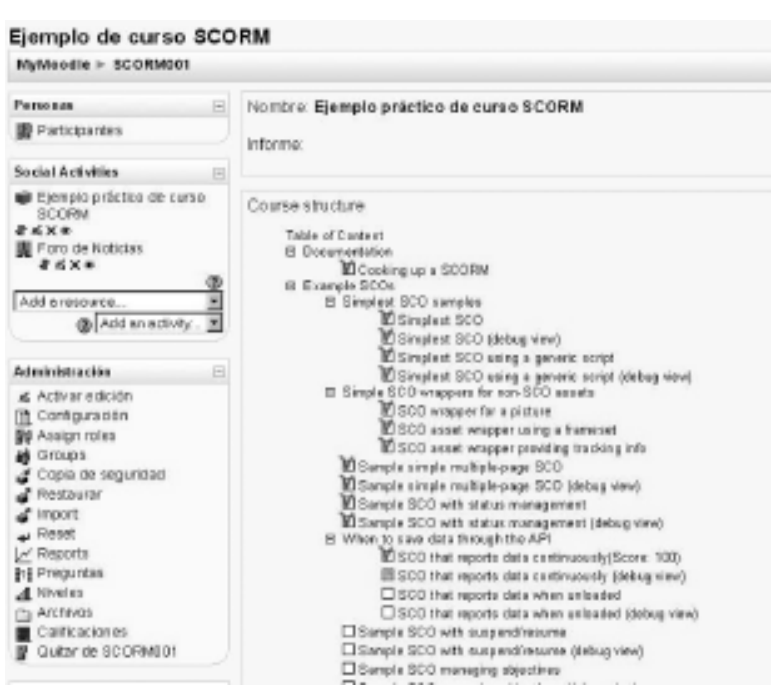

La figura 4 es un ejemplo de cómo Moodle descomprime el paquete SCORM y visualiza su estructura en formato jerárquico, según la definición que el autor del contenido hizo en su momento. Esta misma figura sirve para ilustrar el proceso de comunicación entre contenido y plataforma. Se puede apreciar cómo se almacena información de qué contenidos ha visitado el alumno (marcados en verde), cuál es el contenido en el que se encontraba cuando se desconectó la última vez (marcado en naranja) o la nota obtenida en la realización de un ejercicio (véase "Score: 100").
En la figura 5, se muestra cómo Moodle muestra los contenidos de un curso SCORM. En la parte izquierda de la pantalla, se visualiza la estructura del curso en la que es posible navegar por los distintos elementos. En la parte derecha se visualiza el contenido seleccionado (en el ejemplo, un archivo PDF).

Por último, en la figura 6 se muestra la herramienta calificaciones de Moodle la cual integra información de seguimiento proporcionada por el curso SCORM.

\section{Herramientas para empaquetar cursos SCORM}

Existen varias herramientas de Software Libre que se pueden utilizar para generar paquetes/cursos SCORM. A continuación se comentan brevemente algunas:

- RELOAD (Reusable e.Learning Object Authoring \& Delivering): proyecto que se centra en el desarrollo de un conjunto de herramientas(h) basadas en especificaciones sobre tecnologías aplicadas a la enseñanza. Está gestionada por la Universidad de Bolton (Reino Unido). Los principales objetivos de este proyecto son facilitar la creación, la reutilización y la compartición de objetos de aprendizaje. Reload incluye un conjunto de herramientas entre las que se encuentra un Editor de contenidos con soporte de SCORM 2004, IEEE LOM, IMS Content Package.

- eXe (e.Learning XHTML Editor Project)(i): es una herramienta de autor que permite crear fácilmente contenidos Web que cumplen SCORM $1.2 \mathrm{e}$

Figura 5. Visualizando un curso SCORM en Moodle

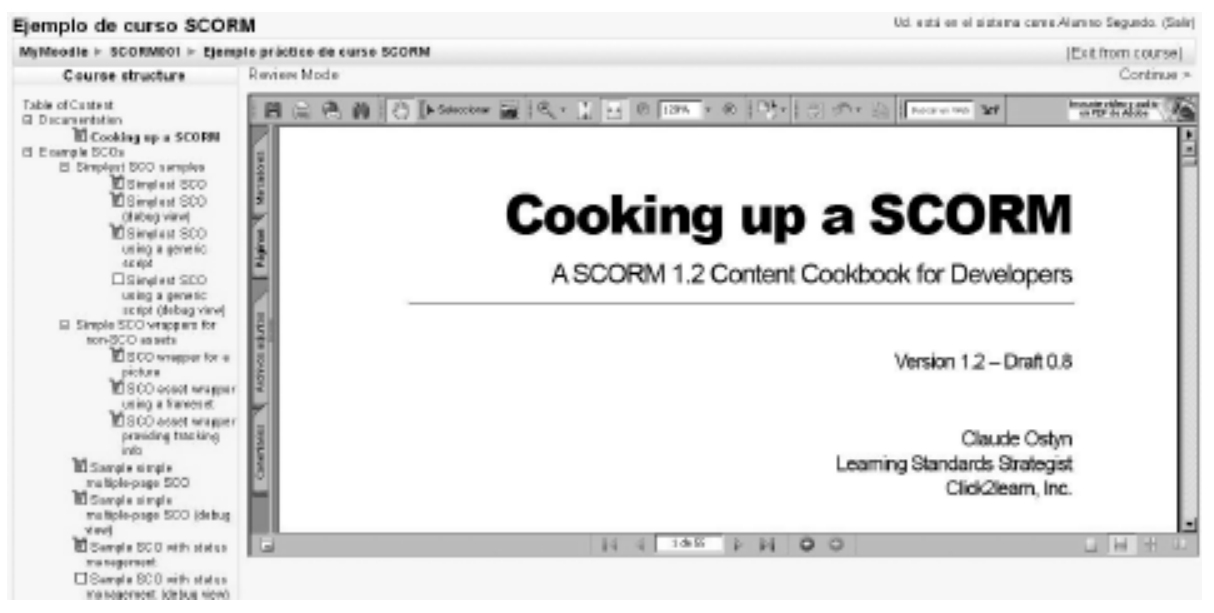


Figura 6. Informe de seguimiento SCORM en Moodle

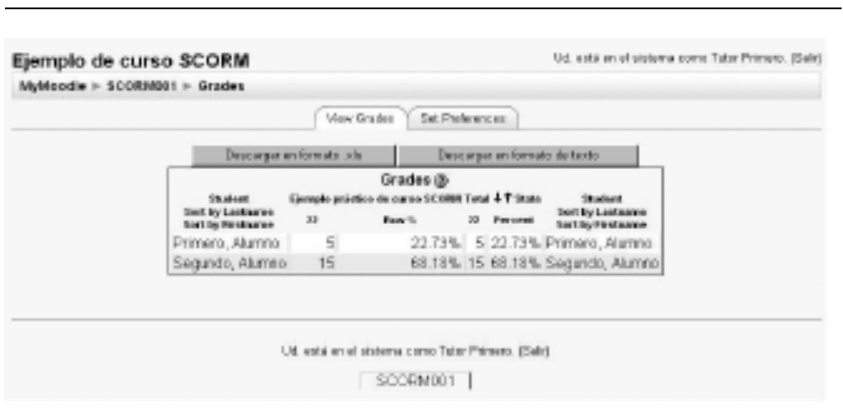

IMS Content Packages. Tiene una gran cantidad de plantillas prediseñadas llamadas iDevices que permiten crear rápidamente ejercicios, incorporar animaciones Flash, editores de texto HTML, poder incluir applets Java, imágenes con texto, incorporar textos procedentes de la Wikipedia, archivos de audio, actividades, etc.

- LAMS (Learning Activity Management System) (j): es una revolucionaria herramienta para diseñar, gestionar y entregar actividades de aprendizaje colaborativas. Permite crear secuencias de actividades de aprendizaje de forma visual. La versión 2.0 incluye soporte para IMS Learning Desing Level A.

\section{CONCLUSIONES}

- Los estándares e.Learning favorecen la reutilización de contenidos con independencia de la plataforma Software sobre la que se soporte, evitando así la dependencia de un fabricante concreto.

- SCORM es un estándar "de facto" que más impacto ha tenido en la industria e.Learning.

- Las distintas versiones de SCORM y la falta de madurez o concreción en algunos aspectos, hacen confusa su implementación desde el punto de vista de las plataformas LMS. Los aspectos no definidos del estándar son interpretados de forma distinta por cada fabricante.

- Se espera que SCORM 2004 3rd Edition sea una evolución estable de SCORM en la que no se prevé grandes cambios en el futuro.

- Existen multitud de aplicaciones de Software
Libre que soportan estándares e.Learning tanto desde el punto de vista de la plataforma LMS como desde las herramientas de generación de contenidos. No es necesario, por tanto, un gran desembolso económico para empezar a generar contenidos e.Learning que cumplan estándares y facilite su intercambio y reutilización.

\section{BIBLIOGRAFÍA}

1. Advanced Distribute Learning. The SCORM Overview [especificación en Internet]. Web de ADL. Disponible en:

http://www.adlnet.gov/scorm/history/12/documents.cfm

2. MACROMEDIA: Getting started with e.Learning standards [en línea] .-Web de Adobe. Disponible en:

http://www.adobe.com/resources/elearning/ standards.html

3. The MASIE Center: Making Sense of Learning: Specifications \& Standards [en línea].-Web de Adobe. Disponible en: http://www.adobe.com/resources/elearning/ standards.html

4. Ostyn, C.: Cooking up a SCORM [en línea].- Web Rustici Software. Disponible en:

http://www.scorm.com/resources/cookbook/

CookingUpASCORM_v1_2.pdf

5. Rebollo, M.: El estándar SCORM para EaD [en línea].-Tesina del Máster "Enseñanza y Aprendizaje Abiertos y a Distancia" de la UNED, 2004. Disponible en:

http://mrebollo.webs.upv.es/pubs/tesina.pdf

\section{Enlaces de interés:}

a) AICC: http://www.aicc.org

b) IMS: http://www.imsglobal.org

c) IEEE LTSC: http://ieeeltsc.org

d) ADL: http://www.adlnet.gov

e) Moodle: http://moodle.org/

f) Moodle Philosophy: http://docs.moodle.org/en/Philosophy

g) Contenido SCORM de ejemplo de Rustici Software: http://www.scorm.com/resources/cookbook/Cooking\%20Up\%2 0a\%20SCORM/CookingUpASCORM_v1_2_Draft_0_8_pif.zip

h) RELOAD Tools: http://www.reload.ac.uk/tools.html

i) eXe Tool: http://eduforge.org/projects/exe/

j) LAMS: http://www.lamsfoundation.org/ 